\title{
Relevance-based processing: Little role for task-relevant expectations
}

\author{
Adam Tapal $^{1}$ (D) $\cdot$ Yaffa Yeshurun $^{2} \cdot$ Baruch Eitam $^{2}$
}

Published online: 3 April 2019

(C) The Psychonomic Society, Inc. 2019

\begin{abstract}
This study examined the role of advance expectations in generating relevance-based selection, using a version of cognitive "blindness" that is driven solely by task relevance. With this irrelevance-induced blindness, participants often fail to report a feature of an irrelevant stimulus, even though the levels of perceptual and cognitive load are minimal (i.e., capacity limitations are not met). Hence, with this phenomenon, selection is based solely on task relevance. In two experiments, we examined such relevance-based selection with a new paradigm in which the participants had to report the location of an object appearing on one of two rings. Critically, while in Experiment 1 the participants could form advance expectations regarding the (ir) relevant stimuli, because the location of the relevant ring and the shape and color of the relevant object were known in advance, in Experiment 2 no concrete advance expectations could be formed. This was established by varying randomly, from trial to trial, the shape, color, and location of relevant and irrelevant stimuli. We found strong irrelevance-induced blindness in both experiments, regardless of whether or not advance expectations were formed. These findings suggest that advance expectations, at least with regard to the task-relevant stimulus' location shape or color, are not necessary for irrelevance-induced blindness to occur; more generally, this implies that such expectations do not play a critical role in selection processes that are based solely on task relevance. We further discuss these findings in the context of Garnerian and Posnerian selection, and their relationship to visual awareness.
\end{abstract}

Keywords Selective attention $\cdot$ Visual awareness $\cdot$ Consciousness $\cdot$ Relevance

Every day, we deal with a vast amount of visual information that we cannot simultaneously process. Luckily, the brain has acquired the capacity to filter much of it. Here we ask: what is necessary for such successful filtering to occur?

Recently, a surprisingly strong version of such filtering that is driven solely by the relevance of the information was documented (Eitam, Yeshurun, \& Hassan, 2013). Unlike most previous demonstrations of so-called blindness (e.g., Most et al., 2001; Rock, Linnett, Grant, \& Mack, 1992; Simons \& Chabris, 1999), in the paradigm used by Eitam et al. (2013), both cognitive and perceptual load were particularly low, ensuring capacity

Electronic supplementary material The online version of this article (https://doi.org/10.3758/s13423-019-01600-1) contains supplementary material, which is available to authorized users.

Baruch Eitam

beitam@psy.haifa.ac.il

Masaryk University, Brno, Czech Republic

2 Department of Psychology, University of Haifa, 31905 Haifa, Israel limitations were not met. In a single-trial experiment, the participants were first asked to concentrate on one of two, differently colored, concentric circles (e.g., "concentrate on the outer circle"). The circles were then presented centrally for a relatively long duration $(500 \mathrm{~ms})$ and without any masking. Following their offset, the participants were surprisingly asked to report the color of both circles. Under these seemingly "loadless" conditions (i.e., two items at the center for $500 \mathrm{~ms}$ with a simple task), strong selection occurred, with up to $25 \%$ of the participants failing to correctly recognize the color of the task-irrelevant circle. Similar relevance-based selection has now been demonstrated using different stimuli and paradigms (Eitam, Shoval, \& Yeshurun; 2015; for a recent review, see Usher, Bronfman, Talmor, Jacobson, \& Eitam, 2018). Because previous blindness demonstrations always involved high load levels (e.g., Most et al., 2001; Rock et al., 1992; Simons \& Chabris, 1999), the findings show that taxing "central" resources in not necessary for such blindness to occur. Hence, it is considered pure relevancebased selection (i.e., the irrelevant information was filtered not because there were no resources to process it, but simply because it was irrelevant). The findings also suggested that specifying in advance the stimulation aspects that are relevant (here, it was the 
circle's spatial location) was sufficient for irrelevance-induced blindness to occur-but is it also necessary?

Even more recently, evidence has accumulated for a second relevance-based selection phenomenon. In a number of studies, Chen, Wyble and colleagues have documented the inability to report features that were apparently necessary to guide selection (e.g., the inability to report the identity of a letter when one's task is to report the location of a letter among distractor digits; Chen \& Wyble, 2015). These authors attributed their finding to forgetting and named it "attribute amnesia" - the rapid forgetting of a (fully) processed stimuli.

The paradigm used by Eitam et al. (2013) and this paradigm differ substantially and may eventually require different explanations. For instance, Chen and Wyble's (2015) task involved a brief $(150 \mathrm{~ms})$, simultaneous presentation of four small peripheral stimuli that were then masked, whereas Eitam et al.'s task involved a central presentation of two larger stimuli for $500 \mathrm{~ms}$ without a mask. Notwithstanding these substantial differences, a recent collaboration (Wyble, Hess, O'Donnell, Chen, \& Eitam, 2018) has seemingly shown that both phenomena are sensitive to a "setting-in" of task expectations. Specifically, irrelevance-induced blindness increased to $63 \%$ when, instead of a single-trial, the surprise question about the irrelevant information arrived after 49 trials in which only the relevant information was probed (i.e., 49 "reportrelevant trials"). Similarly, in a task identical to the one used by Chen and Wyble (2015), participants' failure to report the irrelevant identity of the letter increased from $40 \%$ when probed after a single trial to $70 \%$ after 49 relevant trials.

The fact that the consequences of "set" become more prominent as the expectations regarding the relevance/irrelevance of the upcoming stimulation strengthen further implicated such expectations as a key cause of the observed strong selection. The claim that advanced expectations regarding relevance are critical is also consistent with numerous demonstrations of the potency of prespecifying task relevance to facilitate selection. Specifically, years of research in the field of attention identified at least three aspects of stimulation to which task relevance can be assigned: feature (e.g., Saenz, Buracas, \& Boynton, 2003), object (e.g., Duncan, 1984), and location (e.g., Posner, 1980). This attention-based explanation seemingly nicely accommodates the results of Eitam et al. (2015; Eitam et al., 2013), as in these studies task relevance could be assigned to either location (inner/outer; 2013) or feature (shape/color; 2015). But these conventional loci of selection do not fit the paradigm used by Chen and Wyble (2015), because in that paradigm the location, color, and identity of the target (letter/number) all randomly varied on a trial-by-trial basis. It is impossible, then, to attribute the observed strong selection to prespecification (i.e., expectancies) of a taskrelevant location, feature, or object-simply, because these were all determined only after the array is displayed. Indeed, as stated above, Wyble and colleagues opted for a forgetting-based (rather than encoding-based) explanation.
Importantly, various pieces of evidence suggest that a forgetting-based explanation is not adequate for irrelevancebased blindness (Eitam et al., 2015; Eitam et al., 2013). First, if forgetting was a key factor, one would expect to find an order effect such that reporting the irrelevant stimulus would be worse when probed second (after reporting the relevant stimulus) compared with when it is probed first; yet no such effect was found (Eitam et al., 2013; Wyble et al., 2018; see Experiment 2 of the current study). Second, a recent study (Wyble et al., 2018) found that with the attribute amnesia paradigm, increasing the number of relevant-probed trials before asking about the irrelevant feature was associated with significantly longer response times (RT) to the irrelevant probes (from 15.2 seconds after one trial to 24 seconds after 49 trials). This longer RT suggested a longer search in memory. In contrast, that same study (Wyble et al., 2018) also found that with the irrelevance-induced blindness paradigm, RT was substantially shorter and did not vary significantly with the number of relevant trials ( 4.2 seconds after one trial, 5.4 seconds after 49 trials). This suggested that participants had no reportable trace due to effective filtering, and hence never initiated a search (Wyble et al., 2018). Thus, it seems that a selection-based explanation is more appropriate for pure irrelevance-induced blindness. But what are the underlying mechanisms? Because in our previous studies the relevant aspects of the stimulation (location, color, or shape) were prespecified, advance expectations could be formulated and then serve to filter out irrelevant information (e.g., inhibition; e.g., Cepeda, Cave, Bichot, \& Kim, 1998; Cheal \& Gregory, 1997), to strengthen relevant information (e.g., Bashinski \& Bacharach, 1980; Yeshurun \& Carrasco, 1999), or both. In the current study, we directly test whether such advance expectations are necessary for the emergence of irrelevance-induced blindness.

\section{Experiment 1}

\section{Rationale and method}

The goals of this experiment were twofold. First, to confirm that previous demonstrations of irrelevance-induced blindness can be replicated with the new paradigm employed in this study. Second, it examined whether filtering out (inhibition) of irrelevant information is the dominant mechanism underlying irrelevance-induced blindness. Ninety-eight students (mean age 25.4 years, $S D=3.7 ; 68 \%$ female) from the University of Haifa were seated approximately $60 \mathrm{~cm}$ from the computer screen, in a soundproofed, dimly lit room and were randomly assigned to three conditions. ${ }^{1}$ In two experimental conditions, two rings were presented simultaneously for $500 \mathrm{~ms}$ (see Fig.

\footnotetext{
${ }^{1}$ Here and everywhere, we intended that the number of participants per condition would be similar; the number itself was based on a guesstimate on what would be sufficient to show the effect.
} 
1). In the outer-relevant condition $(N=32)$, the outer ring was designated as relevant, whereas in the inner-relevant condition $(N=32)$, the inner ring was relevant. In the first eight (relevantprobed) trials, a triangle appeared on each ring, and a blue square appeared at the center; all were presented simultaneously for $1,000 \mathrm{~ms}$. The task was to indicate the triangle's location on the ring designated as relevant. This procedure renders the other triangle and ring irrelevant. The purpose of the three conditions were to test whether the irrelevance-stimulus' potential of interference plays any role in relevance-based selection (we found no evidence that it does); our hypothesis was that, if potential for interference does play a role, then more irrelevance-induced blinds should be found in the outerrelevant condition, as participants in that condition should attempt to filter out the inner (more central) regions of the display (where the surprise probe would eventually appear), whereas those in the inner-relevant condition should attempt to filter out the outer (more peripheral) regions of the display (which should not affect processing of the surprise probe). In a third control condition - outer-only $(N=34)$ - a triangle appeared only on the outer ring. Importantly, on the ninth (critical) trial of all the conditions, the square was red. After completing the ninth trial, the participants were surprisingly asked to indicate the square's color in the last trial. If participants in the outer-relevant condition are attempting to filter out the irrelevant, central information, their ability to answer this unexpected question should be worse than that of the participants in the other conditions who either attempted to filter out more peripheral information (inner-relevant condition) or perhaps none at all (outer-only condition). ${ }^{2}$ As mentioned above, no such difference was found. Note that participants who did not correctly indicate the triangle location in the ninth (critical) were removed from the analyzed sample before any inferential statistics were calculated, because failing the task of locating the triangle might be the result of these participants not paying attention to the presented stimuli during the trial.

\section{Results and discussion ${ }^{3}$}

Performance on the relevant ring was high (see Table 1). For attaining better estimates of performance on the task-irrelevant location, we used the levels of blindness found in our previous experiments to set up a Beta $(5.5,1.8)$ prior (see the Supplemental Material for additional information). Table 2 contains the corresponding beta posteriors for each experimental condition and the estimated $q$ parameter (success rate in the surprise question). As can be seen, blindness rates are pronounced in all three conditions (average error rate being

\footnotetext{
${ }^{2}$ Note that evidence of a "doughnut-like" allocation of spatial attention (e.g., Müller \& Hübner, 2002) referred to a mechanism that strengthen the relevant information. To the best of our knowledge, there is no evidence of doughnut-like inhibition.

${ }^{3}$ Scripts and data available from the author upon request (beitam@psy.haifa.ac.il).
}

$\sim 55 \%$ ) and are even larger than those reported by Eitam et al. (2013). No evidence for a difference between the conditions emerged, as the Bayes factors ( $q$ is different vs. $q$ is the same) did not exceed 1 ; they ranged from 0.4 to 0.7 , suggesting that evidence for the null hypothesis is also not substantial. Thus, these finding provide no clear evidence that interference is a necessary factor in irrelevance induced blindness, but the current data cannot rule out the possibility that interference may still modulate the degree of such blindness.

Importantly for our current goals, we are able to replicate irrelevance-induced blindness using yet another paradigm. Like before, here, the participants could develop strong expectations about the target permissible locations (e.g., a target will not appear in the center), showing that blindness emerges when such stable expectancies are present. But the key question we ask in this study is whether such blindness will disappear (or weaken) when such expectancies cannot be formed.

\section{Experiment 2}

\section{Rationale and method}

To test whether advance expectations regarding task-relevant stimuli are necessary for irrelevance-induced blindness, in this experiment we further modified the paradigm to prevent advance formation of expectations about the key-previously established - constituents of advance selection: location, feature, or object. The task and stimuli were similar to the outeronly condition of Experiment 1: No object was present on the inner ring, and an object was always present at the center of the rings. The participants were told that an object will appear on the outer ring and that their task is to report the object's location on the ring. However, unlike in Experiment 1, the task-relevant object, the irrelevant object, and the rings were presented simultaneously. Additionally, the location of the rings varied randomly from trial to trial between four locations, and therefore could not be known in advance. Crucially, the shape and color of the object on the outer ring (the relevant stimulus) and those of the object appearing in the center of the ring (the irrelevant stimulus) also varied randomly (see Fig. 2). Thus, the participants were searching for a random shape, of a random color, in a randomly determined location, while the other objects of the display also randomly varied on these three aspects. Under these conditions, the relevant stimulus is fully defined only once the display is presented and processed (as only then is the ring on which the target appears presented). Hence, if relevance-based selection requires that veridical expectations be in place before the presentation of the stimulation, no such selection should be observed here. In contrast, if selection did occur under the conditions above, the necessity of task-relevant expectations for 


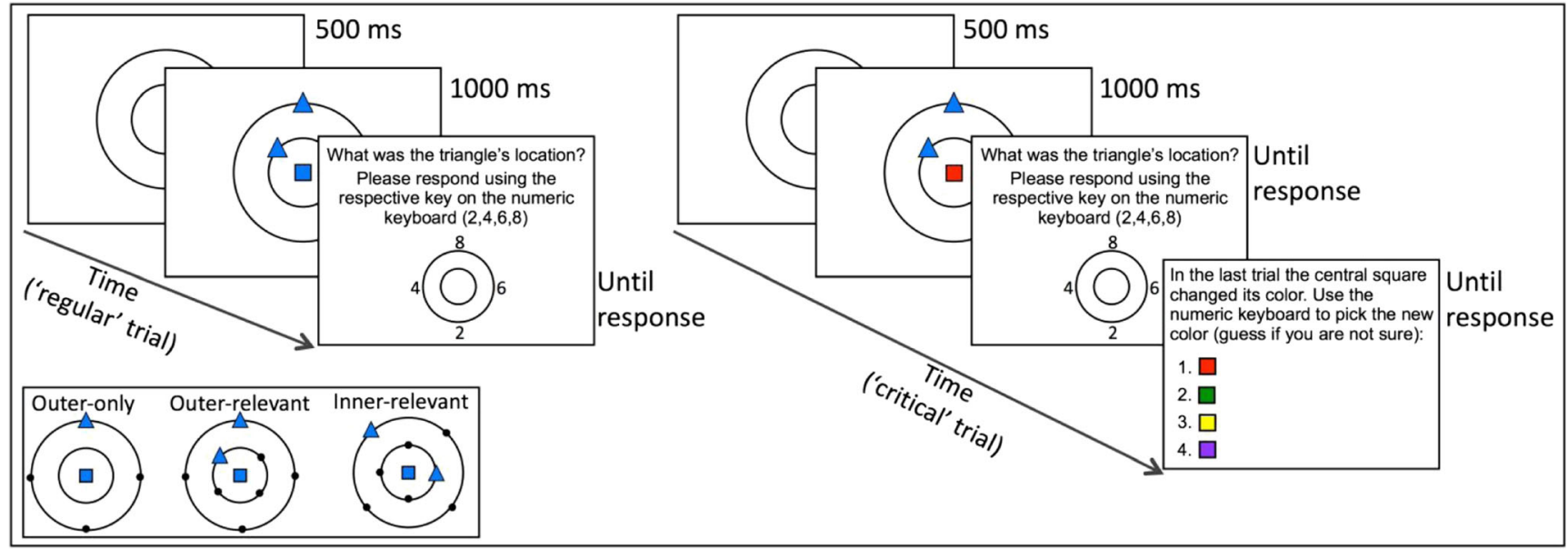

Fig. 1 Schematic of the stimuli and sequence of events in Experiment 1. On the left is an example of a regular trial (one of the initial eight regular trials). On the right is an example of the last (critical) trial. All questions were presented in Hebrew and were displayed until response. The inset depicts examples of the two concentric black rings (diameter: outer $-3.75^{\circ}$; inner $\left.-1.87^{\circ}\right)$ and equilateral triangles $\left(0.5^{\circ}\right)$ in each of the

task relevance-based filtering (at least on these three key parameters) could be rejected.

To enable comparison with Experiment 1, the ninth (critical) trial was identical to the critical trial in the outeronly condition of Experiment 1; that is, on the final trial, all participants saw a (relevant) blue triangle and a (irrelevant) red square. After completing this trial, the participants were asked two surprise questions about the color of (1) the relevant and (2) the irrelevant objects (cf. Chen \& Wyble, 2015). To control for a possible effect of the questions' order, order was counterbalanced such that the

73 students (mean age 25.5 years, $S D=3.8 ; 67 \%$ female) who participated in this experiment were randomly assigned to two conditions: In the relevant-second condition, the participants $(N=37)$ were first asked to indicate the color of the irrelevant object and then the color of the relevant object, whereas in the relevant-first condition $(N=36)$, the questions' order was reversed. Note that regardless of the relevance of the object, the color dimension was always task irrelevant.

Table 1 Performance (\% correct) for stimuli appearing in the taskrelevant ring in Experiments 1-2

\begin{tabular}{llll}
\hline $\begin{array}{l}\text { Trial type } \\
\text { Experiment 1 }\end{array}$ & Outer only & Outer relevant & Inner relevant \\
8 regular trials & $100 \%$ & $95 \%$ & $86 \%$ \\
9th critical trial & $100 \%$ & $94 \%$ & $88 \%$ \\
Experiment 2 & & & \\
& Relevant first & Relevant second & \\
8 regular trials & $99 \%$ & $98 \%$ & \\
9th critical trial & $100 \%$ & $100 \%$ & \\
\hline
\end{tabular}

experimental conditions. Black dots illustrate other possible positions of the triangle on the respective ring, but note that they were not presented during the experiment. The triangle location was chosen randomly. The four optional colors were red, green, yellow, and purple, and they were presented in randomized order. (Color figure online)

\section{Results and discussion}

Here, too, performance on the task-relevant location was high (see Table 1). Like previously, to analyze performance on the task-irrelevant location, a Bayesian betabinomial model was used with a Beta $(5.5,1.8)$ prior. Table 2 contains the corresponding beta posteriors for each question and experimental condition. As can be seen in this table, the blindness rate was again high (average success rate being .23), nominally even greater than in Experiment 1. In fact, for the irrelevant object, the lower end of the credible interval is just above chance level performance (.125), indicating that performance was near complete blindness. Additionally, blindness rates were similar regardless of whether or not the participants first reported the irrelevant color and then the relevant color, or vice versa. Strikingly, the observed blindness occurred regardless of the fact that the color, shape, and location of the stimuli varied randomly, and thus no expectancies about the identity or location could be formed in advance.

Although the participants did have advance knowledge regarding the relative position of the relevant ring (i.e., only the outer ring is relevant), they did not have any advance concrete knowledge of its location. Thus, this finding effectively rules out advance feature-based, object-based, or location-based expectancies as the sole or even necessary factors underlying relevance-based selection.

Interestingly, participants also had rather poor knowledge of the (irrelevant) color of the relevant object (a maximum of $\sim 50 \%$ above chance-level performance, compared with $\sim 87 \%$ above chance for the relevant dimension). We return to this finding in the General Discussion. 
Table 2 Parameters of posterior distributions in Experiments 1-2

\begin{tabular}{|c|c|c|c|c|c|}
\hline Question & Condition & $N$ & $S$ & Posterior & $95 \% \mathrm{CI}^{\dagger}$ for $q$ \\
\hline \multicolumn{6}{|l|}{ Experiment 1} \\
\hline \multirow[t]{3}{*}{ Which color? } & Outer only & $32^{*}$ & 14 & Beta $(19.5,19.8)$ & {$[.34, .65]$} \\
\hline & Outer relevant & 32 & 12 & $\operatorname{Beta}(17.5,21.8)$ & {$[.30, .60]$} \\
\hline & Inner relevant & $28^{*}$ & 15 & Beta $(20.5,14.8)$ & {$[.42, .74]$} \\
\hline \multicolumn{6}{|l|}{ Experiment 2} \\
\hline \multirow[t]{2}{*}{ What was the color of the irrelevant object? } & Relevant second & 37 & 5 & Beta $(10.5,33.8)$ & {$[.13, .37]$} \\
\hline & Relevant first & 36 & 6 & Beta $(11.5,31.8)$ & {$[.15, .41]$} \\
\hline \multirow[t]{2}{*}{ What was the color of the relevant object? } & Relevant second & 37 & 10 & Beta $(15.5,28.8)$ & {$[.22, .49]$} \\
\hline & Relevant first & 36 & 13 & Beta $(18.5,24.8)$ & {$[.29, .58]$} \\
\hline
\end{tabular}

Note. $N=$ the total number of participants; $S=$ the number of participants who answered the surprised question correctly; $q=$ success parameter.

*Participants who provided an incorrect response to the main task in the critical trial were excluded. $\dagger$ Equal-tailed

\section{General discussion}

The two experiments included in this study are a first step toward identifying candidate mechanisms for the recent finding of pure irrelevance-induced blindness and more broadly for relevance-based processing. We begin with the assertion that our experiments involved only minimal perceptual and cognitive load. Hence, in these experiments resources were available for the processing of both relevant and irrelevant information and therefore filtering cannot not be viewed as a passive outcome of resources allocation to the relevant stimuli (cf. Lavie, 1995). It follows from this assertion that the filtering underlying irrelevance-induced blindness is due to an active mechanism(s).

\section{Filtering without expectations?}

Although it is not always made explicit, it is accepted that advance filtering is based on forming expectations about the task relevance of an object, feature, or location - these are the ways in which mechanisms of (selective) attention are thought to be set. Yet the results of Experiment 2 show unequivocally that very strong relevance-based selection occurred even when the participants could not reliably form such expectations. So how can filtering occur without knowing in advance what/where should be filtered? We consider several optional answers to this question: the first is a very rapid filtering occurring poststimulus onset. It is definitely possible that under the high degree of uncertainty that existed in Experiment 2 regarding both targets and distractors, the system shifted to such a form of postonset filtering. This is an intriguing possibility that, as far as we know, has not yet been empirically explored. Such postonset filtering may be unique to highuncertainty environments or may be the usual (remarkable) mechanisms of selection. We speculate that, although possible, postonset filtering out is probably not the explanation for the irrelevance-induces blindness found in Experiment 2. This is because under the conditions of Experiment 2, filtering out (e.g., inhibiting) would necessitate (at the very least) locating the relevant ring for determining the location of the irrelevant one, and only then inhibiting it. Such a process would seemingly be rather slow, which does not seem consistent with the near absence of knowledge regarding the irrelevant dimension of color. That is, assuming that processing of the entire stimulation started as soon as the rings were presented, by the time the irrelevant information was located and inhibition could be applied, some processing of the irrelevant information was already established and should be reflected as partial knowledge. ${ }^{4}$ It seems more plausible to merely locate the relevant object and then "filter it in" (i.e., facilitate its processing). It is also possible that after the onset of the stimuli, both filtering in and filtering out take place. First, immediately after the stimuli appear, the relevant ring is located, and "filtering in" of the object that appears on it kicks in. Then, this filtering in of the relevant information is followed by filtering out of all other, irrelevant, aspects of the stimulation in an attempt to avoid any kind of interference/competition.

A third viable explanation is advance filtering out of the irrelevant dimension, such as color. This alternative is supported by the fact that participants' performance on the color dimension in Experiment 2 was also poor for the relevant object (cf. Chen \& Wyble, 2015; Wyble et al., 2018). Although this possibility has never been fully explored in the context of cognitive blindness, there is a large body of experimental and theoretical work supporting the possibility of the filtering out of a dimension (e.g., Garner, 1970).

\footnotetext{
${ }^{4}$ Unfortunately, in this study response times could not be reliably collected. Future work should use them for addressing this question.
} 


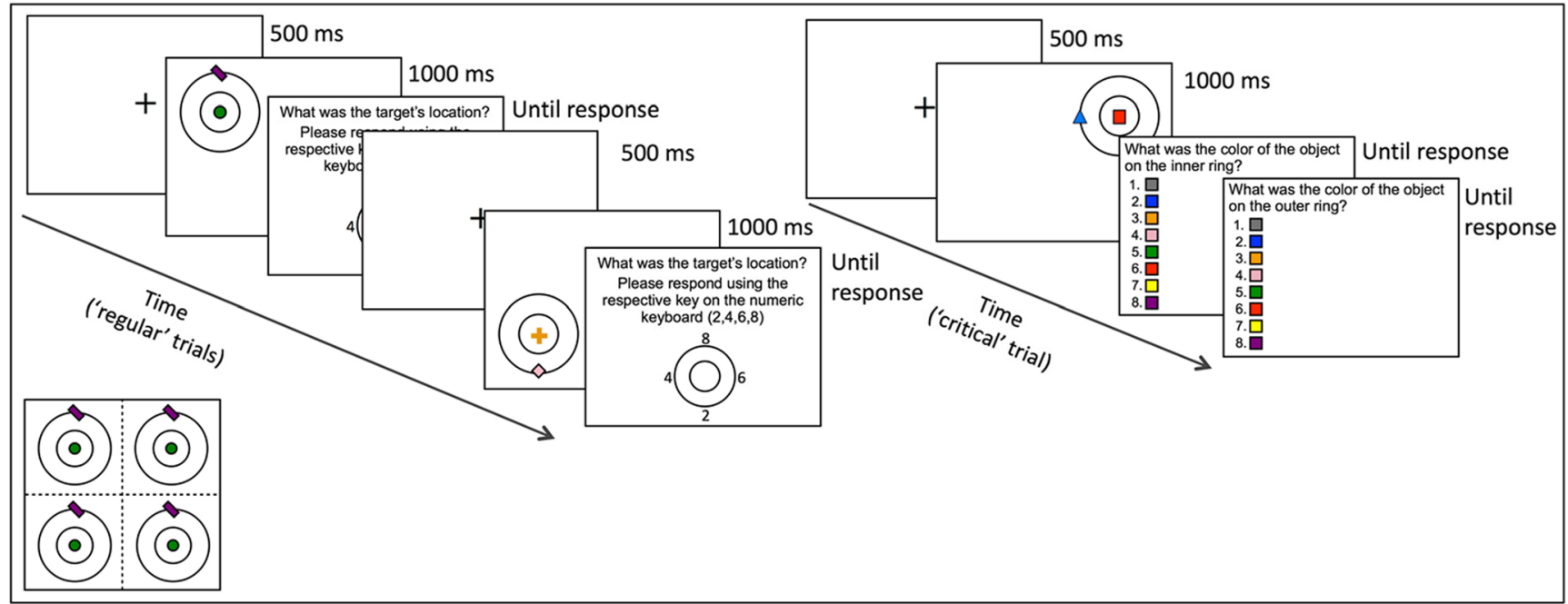

Fig. 2 Schematic of the stimuli and events in Experiment 2. On the left is an example of two regular trials (out of eight regular trials). On these trials, the colors and shapes were chosen randomly from a pool of eight different shapes and colors, with the one constraint being that the two objects never share their shape or color within a given trial. The location of the center of the two rings was also chosen randomly from four possible locations. On the right is an example of the last (critical) trial.

\section{Different types of cognitive "blindness"?}

In fact, Garner's (1970) work on filtering and its relation to separable and integral dimensions may provide completely new directions for the study of the phenomenon of cognitive blindness. That is, according to Garner's framework, we can process the relevant dimension without interference from the irrelevant dimension only if the two dimensions are separable. Hence, if selection is dimension based, this framework predicts that blindness would occur only for separable dimensions.

It is interesting to note that Garner's (1970) original notion of separability was grounded in phenomenal experiencenamely, whether the probed dimensions could vary orthogonally in people's phenomenal experience (Shalev \& Algom, 2000). In Garner's definition, separable dimensions are ones that do not influence the conscious experience of each other; the color of an object, for example, will not consistently influence our conscious experience of its shape. Conversely, integral dimensions will; the hue of an object will influence our experience of its brightness, as these two different dimensions are combined in our conscious experience of color. If indeed, as predicted above, irrelevance-induced blindness disappears for integral dimensions, this would be an empirical link between conscious awareness and (this type of) relevance-based selection. Specifically, it would suggest that dimensional selection, when it occurs, does select out of consciousness (as it is, presumably, possible only when the to-be-selected dimension can be phenomenally differentiated-i.e., consciously perceived as separate - from the irrelevant ones).
Here, eight optional colors were presented (vs. four colors in Experiment 1) to more sensitively measure participants' knowledge of the relevant/ irrelevant color. All questions were presented in Hebrew and appeared until response. The inset depicts the four possible locations for the rings relative to the center of the screen. Only example shapes, colors, and positions on the rings are indicated. (Color figure online)

This state of affairs is different from the previously demonstrated irrelevance-induced blindness (Eitam et al., 2013; Eitam et al., 2015) or the related phenomenon of attribute amnesia (Chen \& Wyble, 2015; Wyble et al., 2018). The latter is argued to stem from rapid forgetting, and the former is thought to be based on so-called Posnerian selection (feature, object, or space based), and for which dimensional separability is irrelevant. Elsewhere (Eitam \& Higgins, 2010, 2016; Usher et al., 2018), it was argued that such Posnerian selection may also stem from the relevance-based activation of knowledge (the mental activation of semantic representations), but not from conscious experience itself (i.e., it does not select out of consciousness). The intriguing possibility that Garnerian selection of dimension does selects out of consciousness deserves further attention.

A final potential explanation is also based on the abovementioned notion that relevance-based effects that are attributed to selective attention reflect selection at the level of knowledge activation rather than consciousness (Eitam \& Higgins, 2010, 2016; Usher et al., 2018). Accordingly, the current results are explained as follows (see a similar explanation for attribute amnesia in Wyble et al., 2018): Because colors are never relevant, the stimuli's colors are never represented, and when participants are (surprisingly) asked to report them, they find no knowledge to report. We find this framework attractive because it can also accommodate findings from very different tasks that the classic selective-attention literature cannot explain, such as relevance-based filtering for semantic/affective priming of attended words (Spruyt, De Houwer, Hermans, \& Eelen, 
2007). Note that it makes different predictions than the Garnerian explanation posited above because it is not sensitive to whether the irrelevant dimension is integral or separable.

To summarize, our findings challenge the role that expectations (regarding location, feature, or object) have been given in selective attention and hence challenge current thoughts about the mechanisms driving relevance-based selection. Much further work is required to understand the specific processes by which this form of pure irrelevance-based selection operates.

Acknowledgements This research was supported by The Israel Science Foundation (ISF) grant number 339/16 and The Binational Science Foundation (BSF) grant number 2016299 to B.E.

\section{References}

Bashinski, H. S., \& Bacharach, V. R. (1980). Enhancement of perceptual sensitivity as the result of selectively attending to spatial locations. Perception \& Psychophysics, 28, 241-248.

Cepeda, N. J., Cave, K. R., Bichot, N. P., \& Kim, M. S. (1998). Spatial selection via feature-driven inhibition of distractor locations. Attention, Perception, \& Psychophysics, 60(5), 727-746.

Cheal, M., \& Gregory, M. (1997). Evidence of limited capacity and noise reduction with single-element displays in the location-cuing paradigm. Journal of Experimental Psychology: Human Perception and Performance, 23(1), 51-71.

Chen, H., \& Wyble, B. (2015). Amnesia for object attributes: Failure to report attended information that had just reached conscious awareness. Psychological Science, 26(2), 203-210.

Duncan, J. (1984). Selective attention and the organization of visual information. Journal of Experimental Psychology: General, 113, 501-517.

Eitam, B., \& Higgins, E. T. (2010). Motivation in mental accessibility: Relevance of a representation (ROAR) as a new framework. Social and Personality Psychology Compass, 4(10), 951-967.

Eitam, B., \& Higgins, E. T. (2016). From reaction ('priming') to motivated selection: Changing conceptualizations of accessibility. Current Opinion in Psychology, 12, 58-62.
Eitam, B., Shoval, R., \& Yeshurun, Y. (2015). Seeing without knowing: Task relevance dissociates between visual awareness and recognition. Annals of the New York Academy of Sciences, 1339(1), 125-137.

Eitam, B., Yeshurun, Y., \& Hassan, K. (2013). Blinded by irrelevance: Pure irrelevance induced "blindness". Journal of experimental psychology: Human perception and performance, 39(3), 611-615.

Garner, W. R. (1970). The stimulus in information processing. American Psychologist, 25, 350-358.

Lavie, N. (1995). Perceptual load as a necessary condition for selective attention. Journal of Experimental Psychology: Human Perception and Performance, 21, 451-468.

Most, S. B., Simons, D. J., Scholl, B. J., Jimenez, R., Clifford, E., \& Chabris, C. F. (2001). How not to be seen: The contribution of similarity and selective ignoring to sustained inattentional blindness. Psychological Science, 12(1), 9-17.

Müller, M. M., \& Hübner, R. (2002). Can the spotlight of attention be shaped like a doughnut? Evidence from steady-state visual evoked potentials. Psychological Science, 13(2), 119-124.

Posner, M. I. (1980). Orienting of attention. Quarterly Journal of Experimental Psychology, 32, 3-25.

Rock, I., Linnett, C. G., Grant, P. P., \& Mack, A.(1992). Perception without attention: Results of a new method. Cognitive Psychology, 24, 502-534.

Saenz, M., Buracas, G. T., \& Boynton, G. M (2003). Global feature-based attention for motion and color. Vision Research, 43, 629-637.

Shalev, L., \& Algom, D. (2000). Stroop and Garner effects in and out Posner's beam: Reconciling two conceptions of attention. Journal of Experimental Psychology: Human Perception and Performance, 26, 997-1017.

Simons, D. J., \& Chabris, C. F. (1999). Gorillas in our midst: Sustained inattentional blindness for dynamic events. Perception, 28(9), $1059-1074$.

Spruyt, A., De Houwer, J., Hermans, D., \& Eelen, P. (2007). Affective priming of nonaffective semantic categorization responses. Experimental Psychology, 54(1), 44-53.

Usher, M., Bronfman, Z., Talmor, S., Jacobson, H., \& Eitam, B. (2018). Consciousness without report: Insights from summary statistics and inattention "blindness." Unpublished manuscript.

Wyble B., Hess M., O’Donnell K., Chen, H., \& Eitam, B. (2018). Learning how to exploit sources of information. Memory \& Cognition https://doi.org/10.3758/s13421-018-0881-x

Yeshurun, Y., \& Carrasco, M. (1999). Spatial attention improves performance in spatial resolution tasks. Vision Research, 39(2), 293-306.

Publisher's note Springer Nature remains neutral with regard to jurisdictional claims in published maps and institutional affiliations. 